\title{
Analysis of the resolution of split-ring metamaterial lenses with application in parallel magnetic resonance imaging
}

Jose M. Algarin, , Manuel J. Freire, , Marcos A. Lopez, , Mikhail Lapine, , Peter M. Jakob, , Volker C. Behr, and , and Ricardo Marqués

Citation: Appl. Phys. Lett. 98, 014105 (2011); doi: 10.1063/1.3533394

View online: http://dx.doi.org/10.1063/1.3533394

View Table of Contents: http://aip.scitation.org/toc/apl/98/1

Published by the American Institute of Physics

\section{Articles you may be interested in}

Nonlinear split-ring metamaterial slabs for magnetic resonance imaging

Applied Physics Letters 98, 133508 (2011); 10.1063/1.3574916

Experimental demonstration of a $\mu=-1$ metamaterial lens for magnetic resonance imaging Applied Physics Letters 93, 231108 (2008); 10.1063/1.3043725

Image acceleration in parallel magnetic resonance imaging by means of metamaterial magnetoinductive lenses

AIP Advances 2, 022136 (2012); 10.1063/1.4723675

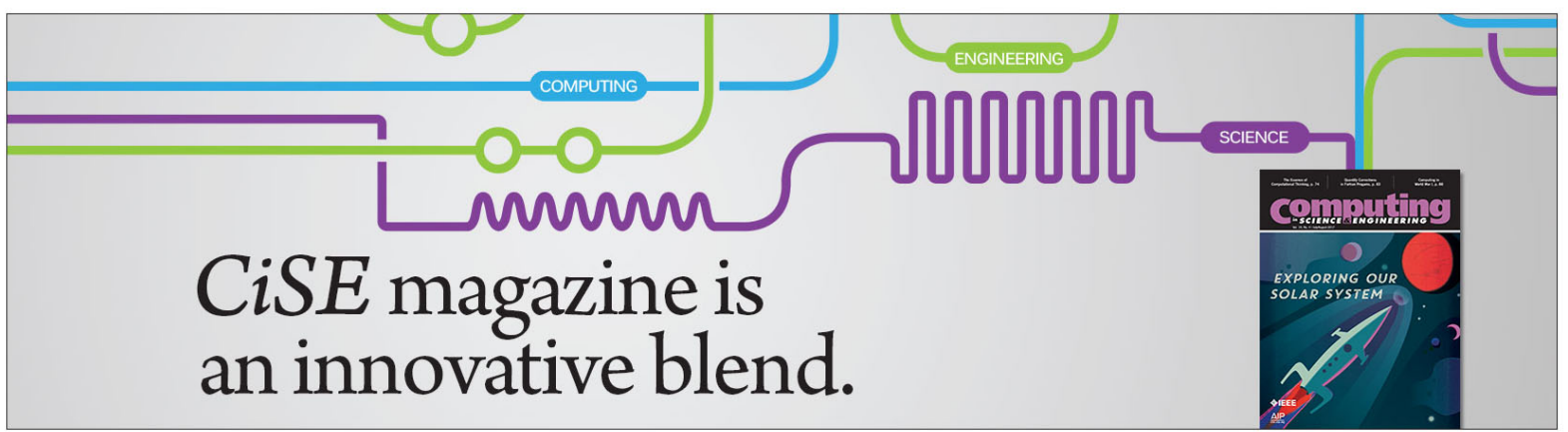




\title{
Analysis of the resolution of split-ring metamaterial lenses with application in parallel magnetic resonance imaging
}

\author{
Jose M. Algarin, ${ }^{1}$ Manuel J. Freire, ${ }^{1, a)}$ Marcos A. Lopez, ${ }^{1}$ Mikhail Lapine, ${ }^{1}$ Peter M. Jakob, ${ }^{2}$ \\ Volker C. Behr, ${ }^{2}$ and Ricardo Marqués ${ }^{1}$ \\ ${ }^{1}$ Department of Electronics and Electromagnetism, Faculty of Physics, University of Seville, \\ Avda. Reina Mercedes s/n, 41012 Sevilla, Spain \\ ${ }^{2}$ Department of Experimental Physics 5 (Biophysics), Institute of Physics, University of Würzburg, \\ Am Hubland, 97074 Würzburg, Germany
}

(Received 8 October 2010; accepted 10 December 2010; published online 6 January 2011)

\begin{abstract}
In this work, we experimentally determine the resolution of split-ring metamaterials lenses with emphasis in magnetic resonance imaging applications. Two small sources are used to determine the minimal resolution of the lens, which is compared with previous theoretical predictions. Taking into account this minimal resolution, a second experiment is designed in order to study the ability of a split-ring lens to improve the localization of the field produced by two closely spaced coils. This ability could find application in parallel magnetic resonance imaging, which take advantage of the distinct coil sensitivities in order to reduce the image acquisition time. (C) 2011 American Institute of Physics. [doi:10.1063/1.3533394]
\end{abstract}

The interesting properties of most metamaterials occur in a very narrow band of frequencies due to the resonant nature of the elements that constitute the periodic structure. This narrow bandwidth is usually cited, in addition to losses, as one of the main limitations for metamaterial applications (see, for instance, Ref. 1 and references therein). However, this narrow-band response is not a big problem for magnetic resonance imaging (MRI) applications, as far as MR images are acquired by measuring radiofrequency (RF) signals inside a relatively narrow frequency band. One of the most striking properties of metamaterials is the ability of a metamaterial slab with relative permittivity $\varepsilon_{r}$ and relative permeability $\mu_{r}$, both equal to -1 , to focus the electromagnetic field into a two-dimensional subwavelength spot, thus behaving as a superlens with subwavelength resolution. ${ }^{2}$ If the frequency of operation is sufficiently low, as it happens in MRI, we are in the realm of the quasistatics, and we only need a metamaterial slab with $\varepsilon_{r}=-1$ or $\mu_{r}=-1$ (depending on the electric or magnetic nature of the quasistatic field) in order to observe these effects. In two previous works, some of the authors studied the applications in MRI of the aforementioned focusing properties of split-ring $\mu_{r}=-1$ metamaterial lenses. It was shown that, in some circumstances, these devices can enhance the sensitivity of a single MRI surface coil, as a consequence of its subwavelength focusing properties.

In this letter, we will specifically analyze the limits for the resolution of $\mu=-1$ split-ring metamaterial lenses, and will explore the applications of such super-resolution in the acquisition of MR medical images. In fact, we feel that super-resolution could find application in parallel MRI (pMRI) techniques, where coil arrays are used to reduce the image acquisition time. ${ }^{5,6}$ pMRI works by taking advantage of the spatially sensitive information inherent in an receiving array of multiple surface coils in order to partially replace time-consuming spatial encoding. For instance, in the PILS technique reported by Griswold et al., ${ }^{5}$ it is assumed that

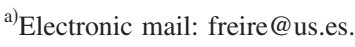

each individual coil in the array has a localized sensitivity pattern. However, this localization takes place only at distances very close to the array because of the spreading out of the magnetic field at farther distances (due to reciprocity, the properties of a detecting coil are directly correlated with the properties of the magnetic field created by the same coil). Since a metamaterial lens can help to discriminate the fields produced by individual coils at deeper distances inside the patient body, this device could be advantageously used in pMRI techniques in order to obtain a better localization of the "field of view" (FOV) of the receiving coils of the array.

It is of general interest for the field of metamaterials and of particular interest for the above application, to determine the minimum distance between sources that can be discriminated by means of a split-ring lens, that is, the minimum resolution of this device. Note that image formation in superlenses substantially differs from image translation in swiss-roll $^{7}$ or wire media ${ }^{8,9}$ endoscopes, where the image is translated pixel-to-pixel from one interface of the device to another. ${ }^{10}$ In these devices, the unit cell size is the natural limit of resolution. In superlenses, however, images are reproduced at some distance from the interfaces through amplification and decay of evanescent modes. ${ }^{2,11,12}$ For this reason, the limits imposed to resolution by the discrete nature of superlenses are not so strong as in endoscopes and may involve several unit cells. The minimum resolution $\Delta$ provided by a homogeneous metamaterial slab of thickness $d$ with negative permittivity and/or permeability is ( $\mathrm{see}^{1}$ and references therein)

$$
\Delta=2 \pi d / \ln (2 / \delta),
$$

where $\delta$ accounts for the losses of the slab, which in the present case would be given by the imaginary part of the complex permeability. In a previous work, ${ }^{3}$ some of the authors reported a split-ring metamaterial lens consisting of a periodic three-dimensional array of capacitively loaded splitring resonators with periodicity $a=1.5 \mathrm{~cm}$ and thickness $d$ $=2 a$. The homogenization of this lens according to Eq. 13 in Ref. 13 predicts a relative complex permeability $\mu_{r}=-1$ 

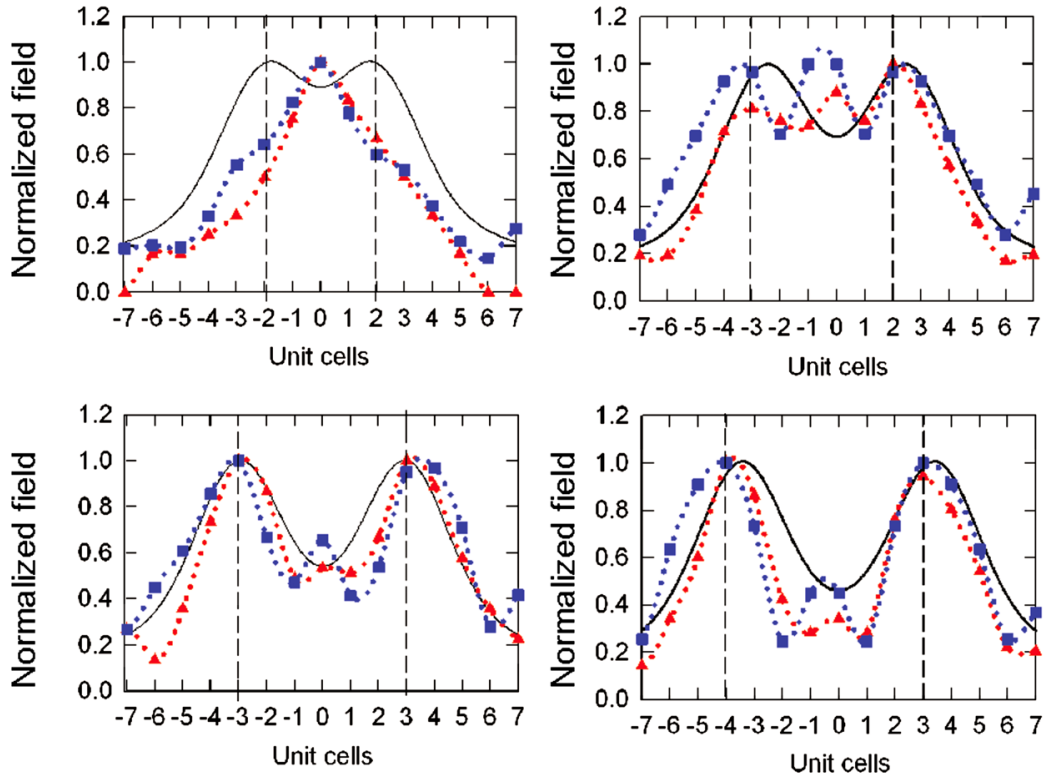

FIG. 1. (Color online) Normalized calculations and measurements of the field produced at the image plane of the lens for several distances between the input antennas: 4, 5, 6, and 7 unit cells. The vertical dashed lines show the relative location of the input antennas. Solid lines: calculations provided by the homogenization model. Red triangles: measurements. Blue squares: calculations provided by the discrete model. $-j 0.25$ at the operating frequency of this device $(63.87 \mathrm{MHz}$, i.e., the Larmor frequency of a $1.5 \mathrm{~T}$ MRI system). Thus, after substituting in the above equation both the thickness $d=2 a$ and the estimated losses $\delta=0.25$, a minimum resolution of $\Delta=6 a$, expressed as a function of the periodicity, is obtained. In the present work, this value is verified by means of the comparison with the results provided by both an experiment and a different theoretical model ${ }^{14}$ which, in the following, will be termed discrete model. The discrete model can be employed to study the electromagnetic properties of finite samples of realistic metamaterials, composed of splitring resonators, and we feel it is an efficient and rigorous approach which takes into account the discrete structure and the finite extent of realistic lenses.

In our experiment, the images generated by the lens reported in Ref. 3 for a pair of small sources are studied. In the experimental setup, a pair of input antennas consisting of copper loops with the same diameter as the rings in the lens is placed at a distance of the lens equal to its thickness (3 $\mathrm{cm})$. The antennas are connected in series, so that currents and excited fields are the same on both antennas. Coaxial cables of $1 \mathrm{~mm}$ in diameter are used to connect the antennas, so that the incident field on the lens is only produced by the antennas and not by the connections. The antennas were placed in front of the lens rings, and the distance between them was always an integer multiple of the lens periodicity. The "image" is obtained at the exit interface of the lens by measuring the transmission coefficient between the input antennas and a small loop probe by means of an Agilent Technologies network analyzer E8363B. For the measurements, the probe is placed at the center of each ring at the exit interface of the lens successively. A matching circuit was included between the port of the network analyzer and the input of the antennas so that the signal in the antennas was well above the background noise. The output signal in the experiments obviously is not a measure of the homogenized field at the exit of the lens, but of the average magnetic flux at each ring of the output interface. However, a comparison between these quantities gives the relative strength of the homogenized field at these locations, and can be used to estimate the resolution. Figure 1 shows the experimental results (red triangles) obtained for input loops separated by 4 ,
5, 6, and 7 unit cells at the frequency of $63.87 \mathrm{MHz}$ for which the best resolution was obtained in the experiments. The vertical dashed lines in the figure show the relative location of the input antennas. In the same figure we show the results provided by both the homogenization model ${ }^{13}$ (black solid line) and the discrete model ${ }^{14}$ (blue squares). The calculations of the homogenization model give the normalized field produced by the input antennas trough an infinite slab of thickness $d=3 \mathrm{~cm}$ and permeability $\mu_{r}=-1-j 0.25$. The results provided by the discrete model ${ }^{14}$ are given by the normalized field produced by the input antennas in the presence of the 2196 rings of the real lens, and the calculation takes into account the coupling between all these rings and the two input antennas. The measurements show that the input antennas can be very well distinguished when they are separated at least a distance of six unit cells $(6 a=9 \mathrm{~cm})$. It can be seen that there is a reasonable agreement between the homogenization model and the experiments when the input antennas were located at a distance of 6 or more lens periods. However, for smaller distances between the antennas, the homogenization model still shows some trace of resolution. The calculations of the discrete model agree much better with the experiments, and also suggest a limit for the lens resolution of six unit cells $(9 \mathrm{~cm})$.

The resolution of a homogeneous metamaterial slab of $\operatorname{Re}\left(\mu_{r}\right)=-1$ only depends on thickness and losses [see Eq. (1)]. However, the resolution of a realistic split-ring metamaterial lens is also limited by the discrete nature of the structure. Our results show that, for the considered losses, both limits agree. They also suggest that, for smaller losses, the limit imposed by the periodicity (six unit cells, for the considered thickness) will prevail. This last result is in agreement with the theoretical predictions of the discrete model. ${ }^{15}$

Once this minimum resolution was determined, we designed an experiment to check the ability of the studied lens to enhance the localization of the FOV of MRI surface coils. From the above results, it is expected that the lens would not be able to enhance the localization of the field produced by coils separated a distance smaller than six unit cells, i.e., 9 $\mathrm{cm}$. To check this, the authors designed an experiment where the lens was combined with different coil arrays of size be- 
(a)

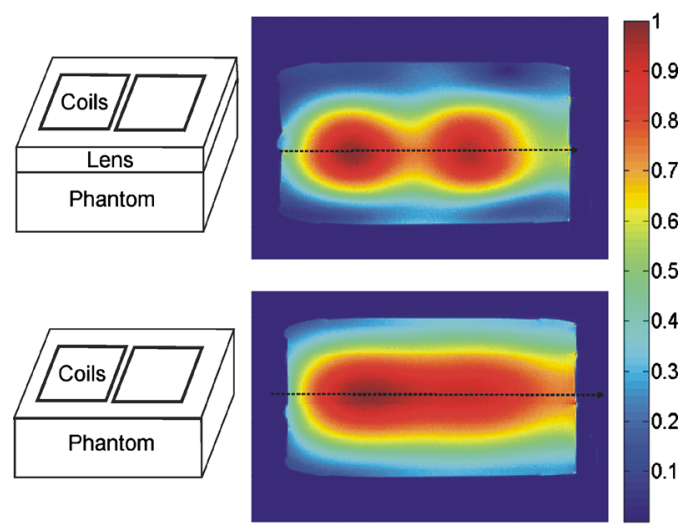

(b)

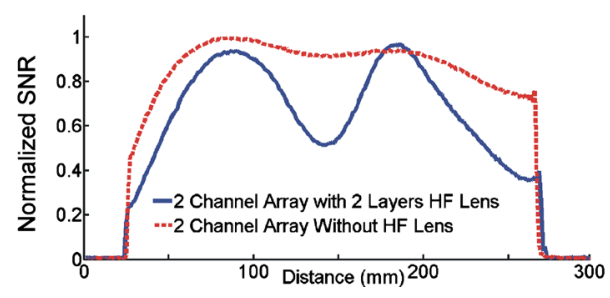

FIG. 2. (Color online) (a) Sketch of the MRI experiment and measurement of the SNR at $6 \mathrm{~cm}$ inside a saline solution phantom in the presence of the lens and in the absence of the lens. (b) Measurement profile along the dashed line shown in a) (blue: with lens, red: without lens).

low and above the value of $9 \mathrm{~cm}$. Experiments done with smaller coils did not show any enhancement of the localization of the FOV, which is in agreement with our previous experiments. However, an enhancement of the FOV was observed when coils of size larger than $9 \mathrm{~cm}$ were used, in particular, a two-channel array of squared coils 12 $\times 12 \mathrm{~cm}^{2}$. As in any MRI array design, each individual element in the array needs to be electrically isolated from the others to minimize the correlated noise. ${ }^{16}$ In the array of squared coils, the elements are decoupled by a shared conductor with a decoupling capacitance; this design yields good MRI sensitivity as well as parallel imaging properties. ${ }^{17}$ Figure 2(a) shows shows a sketch of the two configurations analyzed in the experiment. In one of these configurations, the squared coils are placed on the lens, which is directly placed on a phantom that resembles human tissue. In the other configuration, the coils are directly placed on the phantom. Figure 2(a) also shows the experimental results obtained for both configurations. The measured quantity is the signal to noise ratio (SNR) in a plane parallel to the plane of the array and at a distance inside the phantom of 6 $\mathrm{cm}$ from the surface of the phantom for both configurations. Besides, Fig. 2(b) shows the SNR along the dashed line shown in Fig. 2(a). In general, due to the additional noise introduced by the lens, the SNR measured in the presence of the lens was around $60 \%$ of the SNR measured with the coils directly placed on the phantom. For comparison purposes, the results for the SNR shown in Figs. 2(a) and 2(b) have been normalized to the maximum value in both sets of results. The experiment was carried out in a $1.5 \mathrm{~T}$ Siemens Avanto system (Siemens Medical Systems, Erlangen, Germany) sited at the Department of Experimental Physics 5 (Biophysics) of the University of Würzburg (Würzburg, Germany). The images were acquired using a conventional turbo-spin-echo sequence in both cases. The results show that the field pattern of each coil can be done more independent at a distance where the array by itself cannot. Therefore, the lens provides a better localization of the FOV of each coil. If losses were reduced in the lens in some way, the above considerations on the FOV localization would not change, since for smaller losses the resolution of the lens is mainly limited by its periodicity.

In summary, a simple method for the experimental determination of the minimum resolution of split-ring metamaterial lenses has been presented. The method provides values in good agreement with theoretical predictions. Taking into account the minimum resolution of the analyzed lens, an experiment has been designed in order to show that split-ring metamaterial lenses can improve the field localization of MRI surface coils, a fact that may find applications in pMRI. Nevertheless, since the SNR was degraded by the presence of the lens due to the additional Ohmic losses, in order to achieve a practical application in pMRI, further research aimed to a reduction of these losses must be faced.

This work has been supported by the Spanish Ministerio de Ciencia e Innovación under Project Consolider EMET CSD2008-00066.

${ }^{1}$ R. Marqués, F. Martin, and M. Sorolla, Metamaterials with Negative Parameters: Theory and Microwave Applications (Wiley, Hoboken, New Jersey, 2008).

${ }^{2}$ J. B. Pendry, Phys. Rev. Lett. 85, 3966 (2000).

${ }^{3}$ M. J. Freire, R. Marqués, and L. Jelinek, Appl. Phys. Lett. 93, 231108 (2008).

${ }^{4}$ M. J. Freire, L. Jelinek, R. Marqués, and M. Lapine, J. Magn. Reson. 203, 81 (2010).

${ }^{5}$ M. A. Griswold, P. M. Jakob, M. Nittka, J. W. Goldfarb, and A. Haase, Magn. Reson. Med. 44, 602 (2000).

${ }^{6}$ M. A. Griswold, P. M. Jakob, R. M. Heidemann, M. Nittka, V. Jellus, J. Wang, B. Kiefer, and A. Haase, Magn. Reson. Med. 47, 1202 (2002).

${ }^{7}$ M. C. K. Wiltshire, J. B. Pendry, I. R. Young, D. J. Larkman, D. J. Gilderdale, and J. V. Hajnal, Science 291, 849 (2001).

${ }^{8}$ P. Belov, Y. Hao, and S. Sudhakaran, Phys. Rev. B 73, 033108 (2006).

${ }^{9}$ X. Radu, D. Garray, and C. Craeye, Metamaterials 3, 90 (2009).

${ }^{10}$ R. Syms, E. Shamonina, and L. Solymar, Metamaterials (to be published).

${ }^{11}$ R. Marqués and J. Baena, Microwave Opt. Technol. Lett. 41, 290 (2004).

${ }^{12}$ R. Marqués, M. J. Freire, and J. D. Baena, Appl. Phys. Lett. 89, 211113 (2006)

${ }^{13}$ J. D. Baena, L. Jelinek, R. Marqués, and M. G. Silveirinha, Phys. Rev. A 78, 013842 (2008).

${ }^{14}$ M. Lapine, L. Jelinek, R. Marqués, and M. J. Freire, IET Proc. Microwaves, Antennas Propag. 4, 1132 (2010).

${ }^{15}$ M. Lapine, L. Jelinek, M. J. Freire, and R. Marqués, Phys. Rev. B 82, 165124 (2010).

${ }^{16}$ P. B. Roemer, W. A. Edelstein, C. E. Hayes, S. P. Souza, and O. M. Mueller, Magn. Reson. Med. 16, 192 (1990).

${ }^{17}$ T. Lanz, T. Steinberger, S. Dodd, F. F. Paiva, A. C. Silva, A. P. Koretsky, Proceedings of the 14th ISMRM, Seattle, WA, 6-12 May 2006 (unpublished), p. 2589. 